\title{
Correction: Patterns of opioid prescribing by dentists in a pediatric population: a retrospective observational study
}

Errors occurred in an article published Aug. 6, 2019. ${ }^{1}$

In the section "Dental prescriber characteristics," the sentence "More than half had graduated from dental school before 1991 (Table 1)." should have read "More than a third had graduated from dental school before 1991 (Table 1)."

In the second paragraph of the section "Proportion of dispensed opioids prescribed by dentists," the sentence "Of the total number of licensed dentists in the province during the study period, $17.3 \%-22.2 \%$ prescribed opioids to patients less than 18 years (Table 2)." should have read "Of the total number of licensed dentists in the province during the study period, $12.9 \%-21.6 \%$ prescribed opioids to patients less than 18 years (Table 2)."

In the section "Opioids prescribed for children less than 12 years," the sentence "Very few prescriptions were dispensed to children less than age 12 years (range 42-64 prescriptions per year) (Figure 3)." should have read "Very few prescriptions were dispensed to children less than age 12 years (range 42-70 prescriptions per year) (Figure 3).”
In the second paragraph of the section "Opioid prescribing and dental specialty," the sentence "On a per-prescription basis, general dentists prescribed lower dosages of codeine (mean 34.17 [SD 2.77] MME/d per prescription v. 40.21 [SD 0.84] $\mathrm{MME} / \mathrm{d}$ per prescription) and had more dispensations per patient (mean 1.03 [SD 0.01] v. 1.01 [SD 0.01]) $(p<0.01$, paired $t$ test) than oral surgeons/specialists." should have read "On a per-prescription basis, general dentists prescribed lower dosages of codeine (mean 34.17 [SD 2.77] MME/d per prescription v. 40.21 [SD 0.84] MME/d per prescription) and had more dispensations per patient (mean 1.03 [SD 0.01] v. 1.01 [SD 0.003]) $(p<0.01$, paired $t$ test) than oral surgeons/specialists."

In the second paragraph of the section "Year of graduation and location of dental prescribers," the sentence "Urban dentists wrote the bulk of opioid prescriptions each year of the study (mean 92.6\% [SD 0.76\%]) and wrote more prescriptions per year on an individual basis than rural dental prescribers (mean 24.30 [SD 1.85] v. 14.63 [SD 2.96]) $(p<0.01$, paired $t$ test) (Appendix 2, available at www.cmajopen.ca/

Table 1: Characteristics of licensed dentists in Nova Scotia who prescribed opioid analgesics dispensed to children less than 18 years at community pharmacies

\begin{tabular}{|c|c|c|c|c|c|c|c|}
\hline \multirow[b]{2}{*}{ Characteristic } & \multicolumn{7}{|c|}{ Year; no. (\%) of dental prescribers } \\
\hline & $\begin{array}{l}2011 / 12 \\
n=111\end{array}$ & $\begin{array}{l}2012 / 13 \\
n=117\end{array}$ & $\begin{array}{l}2013 / 14 \\
n=121\end{array}$ & $\begin{array}{c}2014 / 15 \\
n=93\end{array}$ & $\begin{array}{l}2015 / 16 \\
n=101\end{array}$ & $\begin{array}{c}2016 / 17 \\
n=97\end{array}$ & $\begin{array}{c}2017 / 18 \\
n=72\end{array}$ \\
\hline \multicolumn{8}{|l|}{ Location } \\
\hline Urban & $95(85.6)$ & 97 (82.9) & $107(88.4)$ & $81(87.1)$ & $91(90.1)$ & $87(89.7)$ & 65 (90.3) \\
\hline Rural & $16(14.4)$ & $20(17.1)$ & $14(11.6)$ & $12(12.9)$ & $10(9.9)$ & $10(10.3)$ & $7(9.7)$ \\
\hline \multicolumn{8}{|l|}{ Specialty } \\
\hline General dentistry & $86(77.5)$ & $90(76.9)$ & $96(79.3)$ & $67(72.0)$ & $76(75.2)$ & $73(75.3)$ & $46(63.9)$ \\
\hline \multirow[t]{2}{*}{$\begin{array}{l}\text { Oral surgery or other } \\
\text { specialty }\end{array}$} & $25(22.5)$ & $27(23.1)$ & $25(20.7)$ & $26(28.0)$ & $25(24.8)$ & $24(24.7)$ & $26(36.1)$ \\
\hline & \multicolumn{2}{|c|}{$\begin{array}{c}2011 / 12-2012 / 13 \\
n=162\end{array}$} & \multicolumn{2}{|c|}{$\begin{array}{c}2013 / 14-2014 / 15 \\
n=163\end{array}$} & \multicolumn{3}{|c|}{$\begin{array}{c}2015 / 16-2017 / 18 \\
n=170\end{array}$} \\
\hline \multicolumn{8}{|l|}{ Year of graduation } \\
\hline 1990 or earlier & \multicolumn{2}{|c|}{63} & \multicolumn{2}{|c|}{53} & \multicolumn{3}{|c|}{54} \\
\hline 1991-1995 & \multicolumn{2}{|c|}{21} & \multicolumn{2}{|c|}{17} & \multicolumn{3}{|c|}{22} \\
\hline $1996-2000$ & \multicolumn{2}{|c|}{19} & \multicolumn{2}{|c|}{24} & \multicolumn{3}{|c|}{12} \\
\hline 2001-2005 & \multicolumn{2}{|c|}{21} & \multicolumn{2}{|c|}{20} & \multicolumn{3}{|c|}{15} \\
\hline $2006-2010$ & \multicolumn{2}{|c|}{29} & \multicolumn{2}{|c|}{24} & \multicolumn{3}{|c|}{21} \\
\hline 2011-2015 & \multicolumn{2}{|c|}{9} & \multicolumn{2}{|c|}{25} & \multicolumn{3}{|c|}{38} \\
\hline 2016 or later & \multicolumn{2}{|c|}{-} & \multicolumn{2}{|c|}{-} & \multicolumn{3}{|c|}{8} \\
\hline
\end{tabular}


content/7/3/E497/suppl/DC1)." should have read "Urban dentists wrote the bulk of opioid prescriptions each year of the study (mean 92.6\% [SD 0.76\%]) and wrote more prescriptions per year on an individual basis than rural dental prescribers (mean 24.87 [SD 2.44] v. 14.63 [SD 2.96]) $(p<$ 0.01 , paired $t$ test) (Appendix 2, available at www.cmajopen. ca/content/7/3/E497/suppl/DC1)."

In Table 1, the row labels "urban" and "rural" were transposed. The corrected labels are shown here in bold (Table 1).

Several errors and inconsistencies in the data appeared in Table 2. The corrected values are shown here in bold (Table 2).
The published version of Figure 3 was reformatted from the original submitted version and does not accurately reflect the data. The corrected version of Figure 3 is shown here.

These errors have been corrected at cmajopen.ca.

\section{Reference}

1. Matthews DC, Brillant MGS, Jimoh KO, et al. Patterns of opioid prescribing by dentists in a pediatric population: a retrospective observational study. CMAF Open 2019; 7:E497-503.

CMAJ Open 2019. DOI:10.9778/cmajo.20190174

\begin{tabular}{|c|c|c|c|c|c|c|c|}
\hline \multirow[b]{2}{*}{ Patient age, yr } & \multicolumn{7}{|c|}{ Year; no. of dentists who prescribed opioids (\% of all licensed dentists) } \\
\hline & $2011 / 12$ & $2012 / 13$ & $2013 / 14$ & $2014 / 15$ & $2015 / 16$ & $2016 / 17$ & $2017 / 18$ \\
\hline$<12$ & $22(4.1)$ & $21(3.9)$ & $23(4.1)$ & $21(3.9)$ & $22(4.1)$ & $19(3.4)$ & $20(3.6)$ \\
\hline $12-17$ & $103(19.2)$ & $108(19.9)$ & $112(20.0)$ & $85(15.6)$ & $96(17.8)$ & $90(16.2)$ & $65(11.6)$ \\
\hline All $(<18)$ & $111(20.7)$ & $117(21.5)$ & $121(21.6)$ & $93(17.1)$ & $101(18.7)$ & 97 (17.5) & 72 (12.9) \\
\hline
\end{tabular}

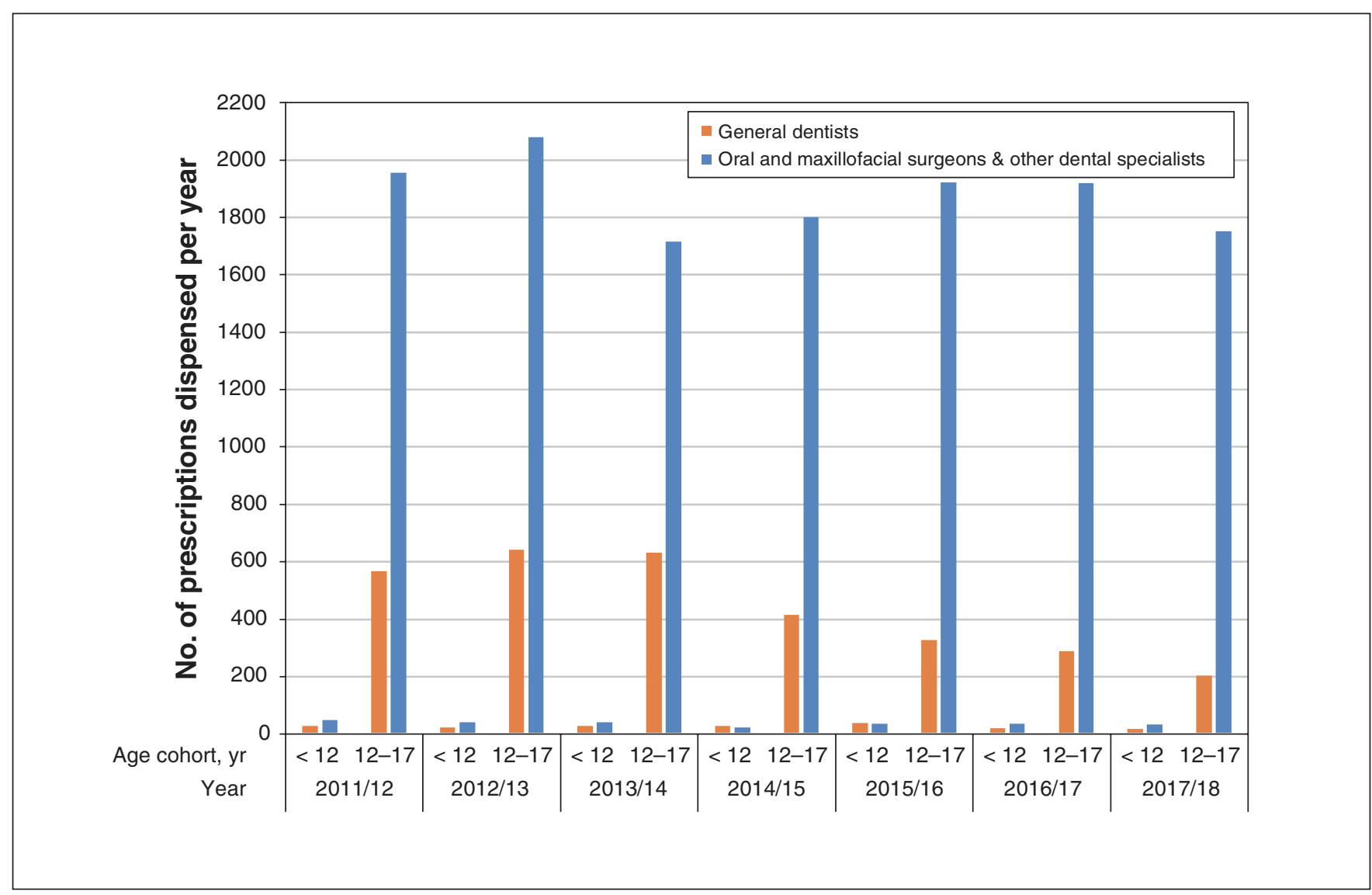

Figure 3: Number of prescriptions for opioid analgesics prescribed by dentists and dispensed to children less than 18 years, by patient age cohort and year dispensed. 